\title{
The MTR A2756G polymorphism is associated with an increase of plasma homocysteine concentration in Brazilian individuals with Down syndrome
}

\author{
J.M. Biselli¹, E.M. Goloni-Bertollo ${ }^{1}$, R. Haddad², M.N. Eberlin² and \\ E.C. Pavarino-Bertelli ${ }^{1}$ \\ ${ }^{1}$ Unidade de Pesquisa em Genética e Biologia Molecular, Departamento de Biologia Molecular, \\ Faculdade de Medicina de São José do Rio Preto, São José do Rio Preto, SP, Brasil \\ ${ }^{2}$ Laboratório ThoMSon de Espectrometria de Massas, Instituto de Química, Universidade Estadual de \\ Campinas, Campinas, SP, Brasil
}

Correspondence to: E.C. Pavarino-Bertelli, UPGEM, FAMERP, Bloco U6, Av. Brigadeiro Faria Lima, 5416, 15090-000 São José do Rio Preto, SP, Brasil

Fax: +55-17-3201-5708. E-mail: erika@famerp.br

\begin{abstract}
Individuals with Down syndrome (DS) present decreased homocysteine (Hcy) concentration, reflecting a functional folate deficiency secondary to overexpression of the cystathionine ß-synthase gene. Since plasma Hcy may be influenced by genetic polymorphisms, we evaluated the influence of C677T and A1298C polymorphisms in the methylenetetrahydrofolate reductase gene (MTHFR), of A2756G polymorphism in the methionine synthase gene (MTR), and of A80G polymorphism in the reduced folate carrier 1 gene on Hcy concentrations in Brazilian DS patients. Fifty-six individuals with free trisomy 21 were included in the study. Plasma Hcy concentrations were measured by liquid chromatography-tandem mass spectrometry with linear regression coefficient $r^{2}=0.9996$, average recovery between 92.3 to $108.3 \%$ and quantification limits of $1.0 \mu \mathrm{mol} / \mathrm{L}$. Hcy concentrations $>15 \mu \mathrm{mol} / \mathrm{L}$ were considered to characterize hyperhomocystinemia. Genotyping for the polymorphisms was carried out by polymerase chain reaction followed by enzyme digestion and allele-specific polymerase chain reaction. The mean Hcy concentration was $5.2 \pm 3.3 \mu \mathrm{mol} / \mathrm{L}$. There was no correlation between Hcy concentrations and age, gender or MTHFR C677T, A1298C and reduced folate carrier 1 A80G genotype. However, Hcy concentrations were significantly increased in the MTR 2756AG heterozygous genotype compared to the MTR 2756AA wild-type genotype. The present results suggest that the heterozygous genotype MTR 2756AG is associated with the increase in plasma Hcy concentrations in this group of Brazilian patients with DS.
\end{abstract}

Key words: Down syndrome; Trisomy 21; Polymorphism; Homocysteine; Mass spectrometry (LC-MS/MS)

Research supported by FAPESP (04/15944-5 and 03/09931-5) and CNPq (302674/2005-5). J.M. Biselli was the recipient of a fellowship from CAPES (CGPP 046/2006).

Received May 29, 2007. Accepted October 18, 2007

\section{INTRODUCTION}

Down syndrome (DS), or trisomy 21 , results from the gene expression of an extra chromosome 21 , which occurs in most cases due to the failure of normal chromosomal segregation during maternal meiosis (1). Individuals with DS present decreased homocysteine (Hcy) concentrations (2). Hcy is an amino acid formed during folate metabolism. It can be remethylated to methionine by the enzyme methionine synthase (MTR), with transfer of the methyl group of methyltetrahydrofolate, formed by the action of the enzyme methylenetetrahydrofolate reductase (MTHFR), or can be converted to cysteine in the transsulfuration pathway, which requires the enzyme cystathionine ß-synthase (CßS) (3). The decreased Hcy con- 
centration observed in individuals with DS is consistent with the location of the CßS gene on chromosome 21 and its overexpression (2) (Figure 1) (4).

Plasma Hcy is influenced by modifiable and non-modifiable factors, such as gender, vitamin status and genetic factors $(5,6)$. The C677T and A1298C polymorphisms of the MTHFR gene are important genetic determinants of Hcy concentrations. The polymorphic alleles result in an enzyme with reduced specific activity $(7,8)$, consequently leading to increased Hcy concentrations $(9,10)$.

Another polymorphism in the gene encoding protein involved in homocysteine-folate metabolism is an adenineto-guanine substitution in nucleotide 2756 of the MTR gene. Some studies $(11,12)$ reported that this polymorphism had an effect in increasing Hcy concentration in the presence of the wild-type allele $(A)$, whereas others $(13,14)$ observed an association between elevated Hcy and this polymorphism in the presence of the mutated allele $(\mathrm{G})$.
However, other studies did not observe any association between this polymorphism and alterations in Hcy concentrations $(15,16)$.

The A80G variant of the reduced folate carrier 1 (RFC1) gene may also contribute to alterations in products derived from this metabolic pathway (11). The RFC1 protein is located in the intestinal mucosa membrane and plays a role in folic acid absorption, transporting 5-methyltetrahydrofolate into the cells (17).

The aim of the present study was to evaluate the influence of the MTHFR C677T, MTHFR A1298C, MTR A2756G, and RFC1 A80G polymorphisms on Hcy concentrations in Brazilian patients with DS.

\section{SUBJECTS AND METHODS}

Fifty-six DS individuals with full trisomy 21 were recruited at the General Genetics Outpatient Service of Hospital de Base, São José do Rio Preto, SP, Brazil, after

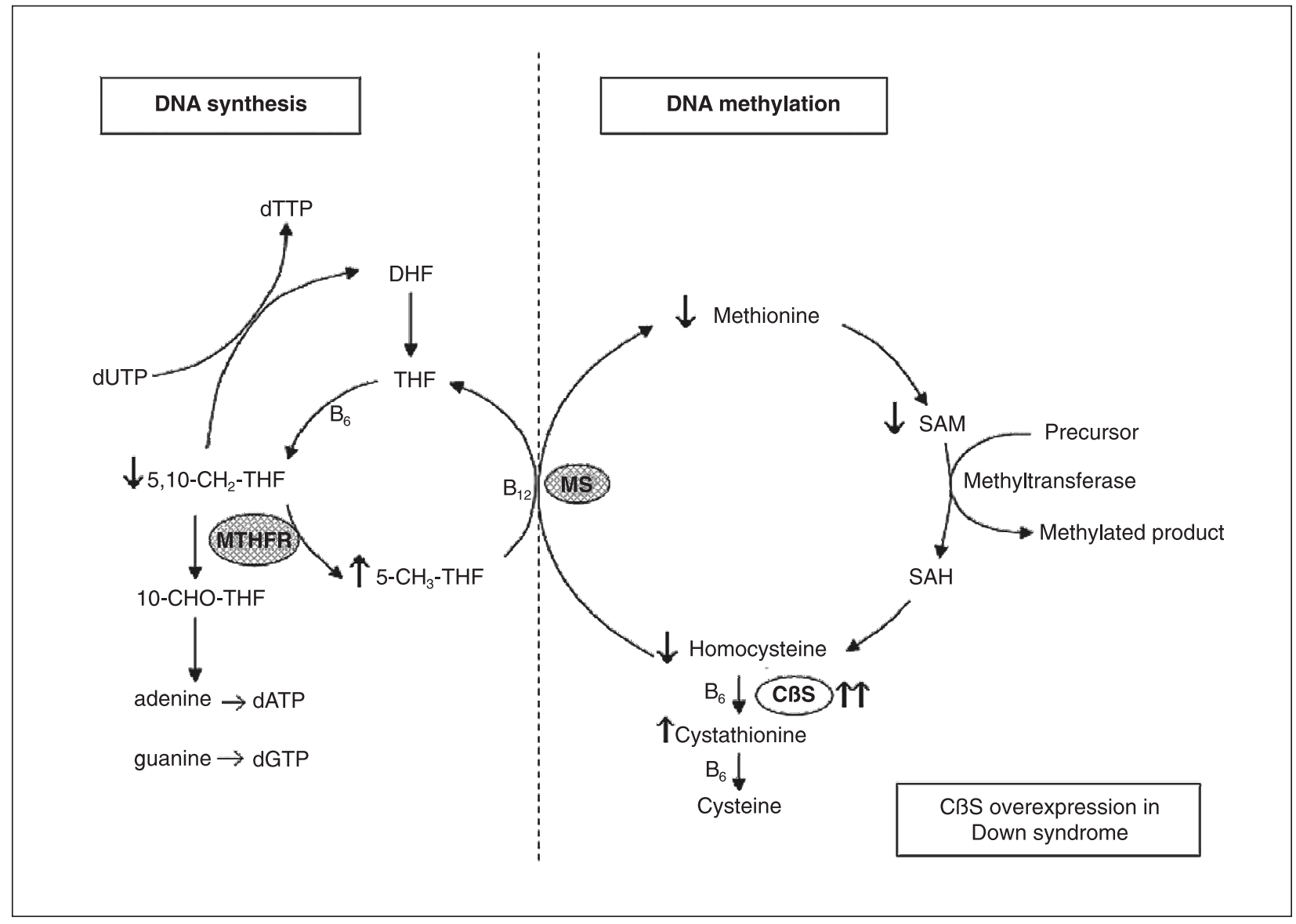

Figure 1. Folate metabolism in Down syndrome (DS) individuals. Arrows indicate direct and indirect alterations in metabolites induced by cystathionine $ß$-synthase (CßS) overexpression in DS individuals (4). dTTP = thymine; dUTP = uracyl; DHF = dihydrofolate; THF $=$ tetrahydrofolate; $5,10-\mathrm{CH}_{2}-\mathrm{THF}=5,10$-methylenetetrahydrofolate; $5-\mathrm{CH}_{3}-\mathrm{THF}=5$-methyltetrahydrofolate; 10-CHO-THF = 10formyl tetrahydrofolate; MTHFR = methylenetetrahydrofolate reductase; MS = methionine synthase; SAM = S-adenosylmethionine; $\mathrm{SAH}=\mathrm{S}$-adenosylhomocysteine. 
obtaining family informed consent.

After an overnight fast, blood samples from all 56 individuals were collected into EDTA-containing tubes, and genomic DNA was extracted from blood leukocytes (18). Polymorphisms were determined by the polymerase chain reaction with upstream and downstream primers flanking the target sequence. Mutation C677T in the MTHFR gene was detected using primer sequences described by Frosst et al. (8), followed by Hinfl digestion. The A1298C polymorphism was determined by allele-specific polymerase chain reaction using primers to separately amplify wild-type and mutated alleles, as follows: forward: 5'- GGA GCT GAC CAG TGA AGA -3', and reverse: 5'- TGT GAC CAT TCC GGT TTG -3', to amplify a 77-base pair (bp) fragment corresponding to allele $A$ (wild type); forward: 5'CTT TGG GGA GCT GAA GGA -3', and reverse: 5'- AAG ACT TCA AAG ACA CTT G -3', to amplify a 120-bp fragment corresponding to allele $\mathrm{C}$ (mutated). A pair of primers was used to amplify a 198-bp segment of the MTHFR gene as positive control of gene amplification (8). Genotyping for the MTR A2756G polymorphism was carried out using specific primers (forward: 5'- CCA GGG TGC CAG GTA TAC AG -3', and reverse: 5'- GCC TTT TAC ACT CCT CAA AAC C -3') to amplify a 498-bp fragment spanning the polymorphism, followed by digestion with enzyme Haell, that digests the fragment in the presence of the mutated $G$ allele. The RFC1 A80G polymorphism was determined as described by Födinger et al. (19). This method for RFC1 A80G genotyping does not distinguish individuals who have one (AAG) or two (AGG) polymorphic alleles, since $D S$ individuals present three alleles for this gene, which is located on chromosome 21.

Blood samples collected into EDTA-containing tubes were centrifuged at $3000 \mathrm{rpm}$ for $10 \mathrm{~min}$, at $18^{\circ} \mathrm{C}$. Plasma was then stored at $-80^{\circ} \mathrm{C}$ until assayed. Total plasma Hcy concentration was measured by mass spectrometry using the Q-TRAP, triple-quadrupole mass spectrometer (Applied Biosystems-Canada-Perkin-Elmer Sciex/QqQ-Trap, Perkin-Elmer Sciex, Thornhill, ON, Canada) using positive electrospray in multiple reaction monitoring mode with internal standard, as previously reported (20-22). Linearity of the method was $r^{2}=0.9996$, average of recovery $101.5 \%$ and quantification limits $1.0 \mu \mathrm{mol} / \mathrm{L}$. Hcy concentrations $>15 \mu \mathrm{mol} / \mathrm{L}$ were considered to characterize hyperhomocystinemia (23).

\section{Statistical analysis}

Data are reported as means $\pm S D$, number or frequency. The distribution of Hcy values in relation to age was analyzed using the Spearman correlation. The $t$-test and ANOVA were employed for the comparisons of Hcy concentrations between genders and between genotypes, respectively, using the logarithmic scale of Hcy concentrations. Hcy concentrations according to the combined MTHFR genotypes (677 and 1298) were evaluated by Mood's median test. MTHFR haplotypes were inferred using the Phase program (version 2.1), which creates population frequency estimates of the haplotypes.

The computer-assisted statistical analyses were carried out with the Minitab for Windows (Release 12.22) program. Hardy-Weinberg equilibrium was determined by the chi-square test using the BioEstat program, except for RFC1 A80G, which presents three alleles in DS individuals. Values of $P \leq 0.05$ were considered to be statistically significant.

\section{RESULTS}

The study group consisted of 56 DS individuals (24 females and 32 males) ranging in age between 1 month and 30 years (mean age: $4.8 \pm 6.7$ years).

The allele frequencies observed were 0.37 for MTHFR 677T, 0.21 for MTHFR 1298C, 0.18 for MTR 2756G, and 0.47 for RFC1 $80 \mathrm{G}$. The allele distribution of the polymorphisms was in Hardy-Weinberg equilibrium $\left(\chi^{2}=0.41\right.$; d.f. $=1 ; \mathrm{P}=0.521$ for MTHFR C677T; $\chi^{2}=3.71$; d.f. $=1 ; \mathrm{P}=$ 0.054 for MTHFR A1298C; $\chi^{2}=1.22$; d.f. $=1 ; P=0.269$ for MTR A2756G).

The MTHFR C677T and A1298C haplotype frequencies inferred by the Phase program were 0.4116 for $\mathrm{C}-\mathrm{A}$, 0.2134 for C-C, 0.3742 for T-A, and 0.0008 for T-C. However, the T-C haplotype was not present in our sample of DS patients. Thus, the T-C frequency in our population was lower than the expected allele combination frequency calculated by the Phase program, confirming negative selection for this haplotype.

The mean Hcy concentration was $5.2 \pm 3.3 \mu \mathrm{mol} / \mathrm{L}$. There was no correlation between Hcy concentrations and age $(\mathrm{Rs}=0.065 ; \mathrm{P}=0.633)$ and the Hcy concentrations did not differ between genders ( $P=0.627$ ).

Mean Hcy concentrations according to genotype for the polymorphisms studied are presented in Table 1. There was no association between Hcy concentrations and genotypes MTHFR C677T $(P=0.110)$, MTHFR A1298C $(P=$ 0.727), RFC1 A80G $(P=0.769)$, or combined MTHFR 677/ 1298 genotypes $(P=0.135)$. However, Hcy concentrations were significantly increased in the MTR 2756AG heterozygous genotype as compared to the MTR 2756AA wild-type genotype $(P=0.025)$.

Statistical analysis was also performed excluding the adults of the sample ( 5 individuals). The mean Hcy concentration was $5.0 \pm 3.3 \mu \mathrm{mol} / \mathrm{L}$. The analysis of the mean Hcy concentrations according to genotype for the polymor- 
Table 1. Plasma homocysteine concentrations according to genotypes MTHFR C677T, MTHFR A1298C, MTR A2756G, and RFC1 A80G in Down syndrome individuals.

\begin{tabular}{lcc}
\hline Genotypes & $\mathrm{N}(\%)$ & Homocysteine $(\mu \mathrm{mol} / \mathrm{L})$ \\
\hline MTHFR C677T & & \\
CC & $23(41.07 \%)$ & $5.83 \pm 4.25$ \\
CT & $24(42.86 \%)$ & $5.14 \pm 2.17$ \\
TT & $3(16.07 \%)$ & $3.59 \pm 2.17$ \\
MTHFR A1298C & & \\
AA & $37(66.07 \%)$ & $5.10 \pm 3.39$ \\
AC & $14(25 \%)$ & $5.49 \pm 3.17$ \\
CC & $5(8.93 \%)$ & $4.86 \pm 3.46$ \\
MTR A2756G & & \\
AA & $39(69.64 \%)$ & $4.71 \pm 2.61$ \\
AG & $14(25 \%)$ & $6.93 \pm 4.51$ \\
GG & $3(5.36 \%)$ & $3.05 \pm 1.13$ \\
RFC1 A80G & & \\
AAA & $10(18.2 \%)$ & $5.92 \pm 5.96$ \\
AG* & $38(69.1 \%)$ & $4.90 \pm 2.74$ \\
GGG & $7(12.7 \%)$ & $5.22 \pm 2.21$ \\
\hline
\end{tabular}

Homocysteine data are reported as means \pm SD. *The genotyping method used in this study does not distinguish AAG from AGG individuals. MTHFR = methylenetetrahydrofolate reductase; $\mathrm{MTR}=$ methionine synthase; RFC1 = reduced folate carrier 1.

phisms studied showed the same results (increased Hcy concentration in the MTR 2756AG genotype) compared for the total group $(P=0.029)$.

Only one individual (a 9-month-old female) presented hyperhomocystinemia $(20.9 \mu \mathrm{mol} / \mathrm{L})$; she was the carrier of a heterozygous genotype for the MTR A2756G polymorphism (MTR 2756AG/MTHFR 677CC/MTHFR 1298AA/ RFC1 80AAA).

\section{DISCUSSION}

Previous studies have suggested that patients with DS have disturbed folate metabolism (2,24). Pogribna et al. (2) reported that the plasma profile of the metabolites involved in the methionine/homocysteine pathway in DS children, including decreased Hcy concentrations, reflect a functional folate deficiency secondary to overexpression of the CßS gene, present on chromosome 21. The increase in the activity of the transsulfuration pathway of Hcy, which results from the overexpression of CßS, may promote a "folate trap" by decreasing the cellular concentration of Hcy and its subsequent remethylation pathway.

Several studies failed to confirm reduced Hcy concentrations in DS patients $(11,25,26)$. Furthermore, in a study with elderly DS patients, Licastro et al. (27) observed that their plasma Hcy was higher and their folate lower than in controls; blood concentrations of vitamin B12 were also lower in DS patients than in controls. The authors attributed most of the cases of mild hyperhomocystinemia in the elderly DS patients to a nutritional folate and B12 deficiency.

In the present study, the mean Hcy concentration of DS patients $(5.2 \pm 3.3 \mu \mathrm{mol} / \mathrm{L})$ was close to that observed by Pogribna et al. (2) in DS children (5.1 $\pm 1.1 \mu \mathrm{mol} / \mathrm{L})$, but lower than the observed by Fillon-Emery et al. (11) (9.8 \pm $0.7 \mu \mathrm{mol} / \mathrm{L})$ and Licastro et al. (27) (12.51 $\pm 1.46 \mu \mathrm{mol} / \mathrm{L})$ in adult and elderly DS patients, respectively, and by Bosco et al. (26) $(8.5 \mu \mathrm{mol} / \mathrm{L})$ in a group of DS patients of various ages (median age: 13 years). This may be due to the fact that in our study only 5 of the 56 DS individuals were adults.

MTHFR is one of the key enzymes in the folate-dependent remethylation of Hcy to methionine. The C677T polymorphism was associated with increased concentrations of Hcy in several studies $(5,9,10,27)$. In DS patients, increased Hcy concentrations were observed in elderly DS subjects with an MTHFR 677TT genotype (27). However, Fillon-Emery et al. (11) found no difference in Hcy concentrations according to the MTHFR C677T genotype in adults with trisomy 21, but observed an increase in Hcy concentrations in the presence of genotypes MTHFR 1298 AA and $\mathrm{AC}$ as compared to the mutated homozygote (CC) in a group of patients taking folic acid supplements. Studies on other populations have not detected any significant differences in Hcy concentrations between A1298C genotypes $(28,29)$. However, elevated Hcy concentrations have been reported for the double heterozygote 677CT/1298AC (30). In our study on DS patients, the Hcy concentrations did not differ among MTHFR genotypes, in agreement with the results of a Brazilian study on healthy children (29). However, the data about the influence of genetic polymorphisms on Hcy concentrations provided by studies with healthy and DS individuals cannot be compared since DS individuals present three copies of the CßS gene, a fact that increases the transsulfuration pathway of Hcy and results in decreased Hcy concentration. Thus, the contribution of genetic polymorphisms to the Hcy concentrations of DS individuals may be underestimated.

The frequency of the MTHFR 677TT genotype observed by us $(16.07 \%)$ was elevated compared to other studies on the Brazilian population ( 10\%) $(29,31)$, but close to those observed by Hobbs et al. (4) (15\%) and Fillon-Emery et al. (11) (12.8\%) in American and French DS patients, respectively. Regarding the MTHFR A1298C polymorphism, the prevalence of homozygous 1298CC individuals in the present study $(8.93 \%)$ was higher than that observed in a large group of healthy Brazilian children 
(5.5\%) (31) but close to the one observed in French DS patients $(7.7 \%)(11)$.

Linkage disequilibrium between MTHFR 677T and $1298 \mathrm{C}$ alleles has been reported $(32,33)$. However, this linkage disequilibrium is not complete since there are studies showing the presence of some individuals with the rare T-C haplotype $(34,35)$. In the present study, no individual with a T-C haplotype was observed, confirming negative selection of this haplotype.

The frequency of the MTR 2756GG genotype (5.36\%) in our DS patients was higher than that observed in French DS patients (2.6\%) (11). The contribution of the MTR A2756G polymorphism to Hcy concentrations has not been fully clarified. This polymorphism has been investigated as a risk factor for some diseases, such as vascular disease, neural tube defects, coronary artery disease and DS, and the evaluation of its influence on Hcy concentrations becomes of great interest. However, most of the studies did not find any relationship between this polymorphism and alterations in Hcy concentrations $(15,16)$, and the few studies that observed this relationship are contradictory with regard to the allele involved. Harmon et al. (12) analyzed the relationship between MTR A2756G genotype and Hcy concentration in a cohort of 625 working men aged 30-49 years and presented evidence that the MTR 2756AA genotype was associated with a modest increase in plasma Hcy concentrations. Similar results were observed in a prospective study of myocardial infarction (36). In a group of DS patients taking folic acid supplements, higher Hcy concentrations were also observed in individuals with the MTR 2756AA genotype compared to the MTR 2756AG genotype (11). On the other hand, Laraqui et al. (13) recently observed a significant contribution of the MTR 2756G allele to a moderate increase in Hcy concentrations in patients with coronary artery disease. In the present study, Hcy concentrations were significantly increased in the MTR 2756AG heterozygous genotype compared to the MTR 2756AA wild-type genotype, in agreement with the observations of Laraqui et al. (13).

Genotype RFC1 80AAA was associated with elevated Hcy concentrations as compared to RFC1 80AG in a group of DS patients using vitamin supplementation (11). The influence of this polymorphism on Hcy concentrations was also observed in healthy individuals, but only when combined with the MTHFR C677T polymorphism (37).

Elevated Hcy concentrations may be harmful since they have been associated with elevated risk of cardiovascular disease, neurodegenerative disorders, psychiatric disorders, and decline of cognitive performance $(13,25,38)$. Guéant et al. (25) observed that a lower intelligence quotient was associated with higher Hcy concentrations in DS patients, and this association was also observed in the presence of the MTHFR 677T allele alone and in combination with the transcobalamin 776G polymorphic allele. However, they did not observe a relationship between intelligence quotient and MTHFR A1298C, MTR A2756G and methionine synthase reductase A66G.

According to Hobbs et al. (4), the presence of genetic variants involved in folate metabolism in DS individuals may confer a survival advantage. These investigators observed a preferential transmission of the MTHFR 677T allele from heterozygous fathers to DS children, and suggested that this may represent a metabolic advantage since the overexpression of the CßS enzyme in these individuals is associated with decreased Hcy, methionine and S-adenosylmethionine concentrations. Hcy is essential for folate-dependent DNA synthesis and for cellular methylation reactions, which are important for fetal development. According to these investigators, a maternal-fetal genotype interaction of the MTHFR C677T polymorphism would also provide a favorable balance of folate distribution between both DNA synthesis and cellular methylation in DS individuals. The preferential transmission of the 677T allele from fathers to DS children was also observed by Rai et al. (39), confirming the hypothesis of Hobbs et al. (4).

In conclusion, our results suggest that the heterozygote genotype MTR 2756AG is associated with the increase in plasma Hcy concentrations in this group of Brazilian patients with DS. However, due to the contradictory results found in different studies, this relationship should be confirmed in a larger sample.

\section{ACKNOWLEDGMENTS}

The authors thank all the participants in this study, the Ding-Down workgroup (multidisciplinary group of health professionals, FAMERP), Prof. Dr. José Antônio Cordeiro for his help with the statistical analysis, and the FAMERP/ FUNFARME.

\section{REFERENCES}

1. Jyothy A, Kumar KS, Mallikarjuna GN, Babu Rao V, Uma Devi B, Sujatha M, et al. Parental age and the origin of extra chromosome 21 in Down syndrome. J Hum Genet 2001; 46: 347-350.

2. Pogribna M, Melnyk S, Pogribny I, Chango A, Yi P, James SJ. Homocysteine metabolism in children with Down syndrome: in vitro modulation. Am J Hum Genet 2001; 69: 8895.

3. Finkelstein JD, Martin JJ. Homocysteine. Int J Biochem Cell Biol 2000; 32: 385-389.

4. Hobbs CA, Cleves MA, Lauer RM, Burns TL, James SJ. Preferential transmission of the MTHFR $677 \mathrm{~T}$ allele to infants with Down syndrome: implications for a survival 
advantage. Am J Med Genet 2002; 113: 9-14

5. Papoutsakis C, Yiannakouris N, Manios Y, Papaconstantinou E, Magkos F, Schulpis KH, et al. The effect of MTHFR(C677T) genotype on plasma homocysteine concentrations in healthy children is influenced by gender. Eur $J$ Clin Nutr 2006; 60: 155-162.

6. Selhub J, Jacques PF, Bostom AG, Wilson PW, Rosenberg $\mathrm{IH}$. Relationship between plasma homocysteine and vitamin status in the Framingham study population. Impact of folic acid fortification. Public Health Rev 2000; 28: 117-145.

7. Weisberg I, Tran P, Christensen B, Sibani S, Rozen R. A second genetic polymorphism in methylenetetrahydrofolate reductase (MTHFR) associated with decreased enzyme activity. Mol Genet Metab 1998; 64: 169-172.

8. Frosst $\mathrm{P}$, Blom HJ, Milos R, Goyette $\mathrm{P}$, Sheppard $\mathrm{CA}$, Matthews RG, et al. A candidate genetic risk factor for vascular disease: a common mutation in methylenetetrahydrofolate reductase. Nat Genet 1995; 10: 111-113.

9. Ulvik A, Ueland PM, Fredriksen A, Meyer K, Vollset SE, Hoff $G$, et al. Functional inference of the methylenetetrahydrofolate reductase $677 \mathrm{C}>\mathrm{T}$ and 1298A $>\mathrm{C}$ polymorphisms from a large-scale epidemiological study. Hum Genet 2007; 121: $57-64$.

10. Castro R, Rivera I, Ravasco P, Jakobs C, Blom HJ, Camilo $\mathrm{ME}$, et al. 5,10-Methylenetetrahydrofolate reductase $677 \mathrm{C}$ $\rightarrow \mathrm{T}$ and 1298A $\rightarrow \mathrm{C}$ mutations are genetic determinants of elevated homocysteine. QJM 2003; 96: 297-303.

11. Fillon-Emery $\mathrm{N}$, Chango A, Mircher $\mathrm{C}$, Barbe $\mathrm{F}$, Blehaut $\mathrm{H}$, Herbeth $B$, et al. Homocysteine concentrations in adults with trisomy 21: effect of $B$ vitamins and genetic polymorphisms. Am J Clin Nutr 2004; 80: 1551-1557.

12. Harmon DL, Shields DC, Woodside JV, McMaster D, Yarnell JW, Young IS, et al. Methionine synthase D919G polymorphism is a significant but modest determinant of circulating homocysteine concentrations. Genet Epidemiol 1999; 17: 298-309.

13. Laraqui A, Allami A, Carrie A, Coiffard AS, Benkouka F, Benjouad $A$, et al. Influence of methionine synthase (A2756G) and methionine synthase reductase (A66G) polymorphisms on plasma homocysteine levels and relation to risk of coronary artery disease. Acta Cardiol 2006; 61: 5161.

14. Feix A, Fritsche-Polanz R, Kletzmayr J, Vychytil A, Horl $\mathrm{WH}$, Sunder-Plassmann $\mathrm{G}$, et al. Increased prevalence of combined MTR and MTHFR genotypes among individuals with severely elevated total homocysteine plasma levels. Am J Kidney Dis 2001; 38: 956-964.

15. Klerk M, Lievers KJ, Kluijtmans LA, Blom HJ, den Heijer M, Schouten EG, et al. The $2756 \mathrm{~A}>\mathrm{G}$ variant in the gene encoding methionine synthase: its relation with plasma homocysteine levels and risk of coronary heart disease in a Dutch case-control study. Thromb Res 2003; 110: 87-91.

16. Kluijtmans LA, Young IS, Boreham CA, Murray L, McMaster $\mathrm{D}, \mathrm{McNulty} \mathrm{H}$, et al. Genetic and nutritional factors contributing to hyperhomocysteinemia in young adults. Blood 2003; 101: 2483-2488.

17. Nguyen TT, Dyer DL, Dunning DD, Rubin SA, Grant KE, Said HM. Human intestinal folate transport: cloning, expression, and distribution of complementary RNA. Gastroenterology 1997; 112: 783-791.

18. Abdel-Rahman SZ, Nouraldeen AM, Ahmed AE. Molecular interaction of [2,3-14C] acrylonitrile with DNA in gastric tissue of rat. J Biochem Toxicol 1994; 9: 191-198.

19. Födinger M, Dierkes J, Skoupy S, Rohrer C, Hagen W, Puttinger $\mathrm{H}$, et al. Effect of glutamate carboxypeptidase II and reduced folate carrier polymorphisms on folate and total homocysteine concentrations in dialysis patients. J Am Soc Nephrol 2003; 14: 1314-1319.

20. de Andrade CR, Fukada SY, Olivon VC, de Godoy MA, Haddad R, Eberlin MN, et al. Alpha1D-adrenoceptor-induced relaxation on rat carotid artery is impaired during the endothelial dysfunction evoked in the early stages of hyperhomocysteinemia. Eur J Pharmacol 2006; 543: 83-91.

21. Haddad R, Mendes MA, Hoehr NF, Eberlin MN. Amino acid quantitation in aqueous matrices via trap and release membrane introduction mass spectrometry: homocysteine in human plasma. Analyst 2001; 126: 1212-1215.

22. Vellasco AP, Haddad R, Eberlin MN, Hoehr NF. Combined cysteine \& homocysteine quantitation in plasma by trap and release membrane introduction mass spectrometry. Analyst 2002; 127: 1050-1053.

23. Measurement and use of total plasma homocysteine. American Society of Human Genetics/American College of Medical Genetics Test and Transfer Committee Working Group. Am J Hum Genet 1998; 63: 1541-1543.

24. Ueland PM, Refsum H, Christensen B. Methotrexate sensitivity in Down's syndrome: a hypothesis. Cancer Chemother Pharmacol 1990; 25: 384-386.

25. Guéant JL, Anello G, Bosco P, Gueant-Rodriguez RM, Romano A, Barone C, et al. Homocysteine and related genetic polymorphisms in Down's syndrome IQ. J Neurol Neurosurg Psychiatry 2005; 76: 706-709.

26. Bosco P, Gueant-Rodriguez RM, Anello G, Barone C, Namour $F$, Caraci $F$, et al. Methionine synthase (MTR) 2756 $(A \rightarrow G)$ polymorphism, double heterozygosity methionine synthase $2756 \mathrm{AG} /$ methionine synthase reductase (MTRR) $66 \mathrm{AG}$, and elevated homocysteinemia are three risk factors for having a child with Down syndrome. Am J Med Genet A 2003; 121: 219-224.

27. Licastro F, Marocchi A, Penco S, Porcellini E, Lio D, Dogliotti G, et al. Does Down's syndrome support the homocysteine theory of atherogenesis? Experience in elderly subjects with trisomy 21. Arch Gerontol Geriatr 2006; 43: 381-387.

28. Narayanan S, McConnell J, Little J, Sharp L, Piyathilake CJ, Powers $\mathrm{H}$, et al. Associations between two common variants C677T and A1298C in the methylenetetrahydrofolate reductase gene and measures of folate metabolism and DNA stability (strand breaks, misincorporated uracil, and DNA methylation status) in human lymphocytes in vivo. Cancer Epidemiol Biomarkers Prev 2004; 13: 1436-1443.

29. Alessio AC, Annichino-Bizzacchi JM, Bydlowski SP, Eberlin MN, Vellasco AP, Hoehr NF. Polymorphisms in the methylenetetrahydrofolate reductase and methionine synthase reductase genes and homocysteine levels in Brazilian children. Am J Med Genet A 2004; 128: 256-260.

30. van der Put NM, Gabreels F, Stevens EM, Smeitink JA, Trijbels FJ, Eskes TK, et al. A second common mutation in the methylenetetrahydrofolate reductase gene: an additional risk factor for neural-tube defects? Am J Hum Genet 1998; 62: 1044-1051.

31. Arruda VR, Siqueira LH, Goncalves MS, von Zuben PM, Soares MC, Menezes R, et al. Prevalence of the mutation 
$\mathrm{C} 677 \rightarrow \mathrm{T}$ in the methylene tetrahydrofolate reductase gene among distinct ethnic groups in Brazil. Am J Med Genet 1998; 78: 332-335.

32. Stegmann K, Ziegler A, Ngo ET, Kohlschmidt N, Schroter B, Ermert A, et al. Linkage disequilibrium of MTHFR genotypes $677 \mathrm{C} / \mathrm{T}-1298 \mathrm{~A} / \mathrm{C}$ in the German population and association studies in probands with neural tube defects (NTD). Am J Med Genet 1999; 87: 23-29.

33. Shi M, Caprau D, Romitti P, Christensen K, Murray JC. Genotype frequencies and linkage disequilibrium in the $\mathrm{CEPH}$ human diversity panel for variants in folate pathway genes MTHFR, MTHFD, MTRR, RFC1, and GCP2. Birth Defects Res A Clin Mol Teratol 2003; 67: 545-549.

34. Scala I, Granese B, Sellitto M, Salome S, Sammartino A, Pepe A, et al. Analysis of seven maternal polymorphisms of genes involved in homocysteine/folate metabolism and risk of Down syndrome offspring. Genet Med 2006; 8: 409-416.

35. Parle-McDermott A, Mills JL, Kirke PN, O'Leary VB, Swanson DA, Pangilinan F, et al. Analysis of the MTHFR 1298A $\rightarrow \mathrm{C}$ and $677 \mathrm{C} \rightarrow$ T polymorphisms as risk factors for neural tube defects. J Hum Genet 2003; 48: 190-193.

36. Chen J, Stampfer MJ, Ma J, Selhub J, Malinow MR, Hennekens $\mathrm{CH}$, et al. Influence of a methionine synthase (D919G) polymorphism on plasma homocysteine and folate levels and relation to risk of myocardial infarction. Atherosclerosis 2001; 154: 667-672.

37. Chango A, Emery-Fillon N, de Courcy GP, Lambert D, Pfister M, Rosenblatt DS, et al. A polymorphism (80G $\rightarrow A$ ) in the reduced folate carrier gene and its associations with folate status and homocysteinemia. Mol Genet Metab 2000; 70: 310-315.

38. Mattson MP, Kruman II, Duan W. Folic acid and homocysteine in age-related disease. Ageing Res Rev 2002; 1: 95111.

39. Rai AK, Singh S, Mehta S, Kumar A, Pandey LK, Raman R. MTHFR C677T and A1298C polymorphisms are risk factors for Down's syndrome in Indian mothers. J Hum Genet 2006; 51: 278-283. 